\title{
Growth Kinetics of Monodisperse Polystyrene Microspheres Prepared by Dispersion Polymerization
}

\author{
Fan Li, ${ }^{1,2}$ Chong Geng, ${ }^{1}$ and Qingfeng Yan ${ }^{1}$ \\ ${ }^{1}$ Department of Chemistry, State Key Laboratory of New Ceramics and Fine Processing, Tsinghua University, Beijing 100084, China \\ ${ }^{2}$ Department of Material Science and Engineering, Tsinghua University, Beijing 100084, China \\ Correspondence should be addressed to Qingfeng Yan; yanqf@mail.tsinghua.edu.cn
}

Received 26 March 2013; Accepted 26 May 2013

Academic Editor: Alain Durand

Copyright (c) 2013 Fan Li et al. This is an open access article distributed under the Creative Commons Attribution License, which permits unrestricted use, distribution, and reproduction in any medium, provided the original work is properly cited.

\begin{abstract}
Dispersion polymerization has been widely applied to the synthesis of monodisperse micron-sized polymer colloidal spheres. Many efforts have been devoted to studying the influence of initial conditions on the size and uniformity of the resultant microspheres, aiming to synthesize micron-size monodisperse colloidal spheres. However, the inner contradiction between the size and the size distribution of colloidal spheres hinders the realization of this goal. In this work, we drew our attention from the initial conditions to the growth stage of dispersion polymerization. We tracked the size evolution of colloidal sphere during the dispersion polymerization, through which we established a kinetic model that described the relationship between the monomer concentration and the reaction time. The model may provide a guideline to prepare large polymer colloidal spheres with good monodispersity by continuous monomer feeding during the growth stage to maintain the concentration of monomer at a constant value in a dispersion polymerization process.
\end{abstract}

\section{Introduction}

As a new functional macromolecule material, monodisperse polymer microspheres have many applications in environmental conservation, biomedicine, colloid science, electronic information material, and many other areas [1-3] due to their superiority of good sphericity, size tunability, large specific surface area, and excellent absorbability [4-6]. Particularly, monodisperse micron-sized spheres are ideal materials as advanced coatings [7], fillers of chromatographic column $[8,9]$, standard particles of electron microscope and Coulter particle size testers, and spacers in LCD [10], which has drawn increasing interest to synthesize monodisperse micron-sized spheres $[11,12]$.

The first report on monodisperse polymer colloidal spheres was the polystyrene spheres prepared by Vanderhoff and Brandford [13]. So far, many synthesis strategies have been developed such as emulsion polymerization, suspension polymerization, dispersion polymerization, and seed swelling polymerization. In view of the demand of micron size and monodispersity, the former two methods are excluded because they cannot meet the two requirements at the same time. Emulsion polymerization can only gain monodisperse colloidal spheres with the size below $1 \mu \mathrm{m}$. Suspension polymerization may achieve large microspheres with size ranging from $10 \mu \mathrm{m}$ to $100 \mu \mathrm{m}$ while the microspheres possess a bad monodispersity [14]. The latter two methods are suitable for the synthesis of uniform colloidal spheres with micron size. Seed swelling polymerization, including the dynamics swelling developed by Okubo et al. [15] and twostep swelling initiated by Ugelstad et al. [16], can attain colloidal spheres with larger sphere diameter. However, in terms of the whole process, seed swelling polymerization is complex. In addition, the monodispersity is determined not only by the swelling process itself, but also by the initial monodispersity of the seeds prepared by dispersion polymerization in advance. Thus, the key point is how to gain larger microspheres with uniform size by dispersion polymerization.

To attain larger microspheres with excellent monodispersity, many efforts have been made to explore the influence of the initial condition of the reaction system on 
the resultant microspheres, such as the dosage of monomer, initiator, and dispersing agent. Generally, with the increase in the monomer's initial concentration, the sphere diameter increases and the distribution of sphere diameter broadens. Thus, there is a suitable range for monomer's initial concentration to achieve uniform and large microspheres $[17,18]$. Similarly, increasing the concentration of initiator leads to higher reaction rate and larger sphere diameter while it may also broaden the distribution of the sphere diameter simultaneously $[17,19,20]$. In addition, the dispersing agent which prevents microspheres from gelatinization and stabilizes the reaction system requires a sufficient supply to guarantee its functions. However, the increase in the amount of the dispersing agent results in a smaller size despite a better monodispersity [21-24]. In this view, the inner contradiction between the size and the size distribution of microspheres hinders the further increase of the size of monodisperse microspheres. It is thus believed that the inner contradiction cannot be solved solely by adjusting the initial conditions.

Recently, Song et al. [25] employed two-stage dispersion polymerization to synthesize large and uniform dyecontained polymer spheres. In this method, the dyecomonomer, which significantly destroyed the final monodispersity if fed into the reaction at the beginning, was added to the reaction system after the nucleation stage. Through this strategy, the influence of the dye-comonomer on the nucleation was avoided and a narrow size distribution of the final colloidal spheres was guaranteed. It was expected that two-stage or multistage dispersion polymerization strategy paved a way to the synthesis of micron-sized large and monodisperse polymer colloidal spheres. However, when and how to feed the extra monomer into the reaction system to achieve this goal remains debatable since most previous works were mainly based on trial and error.

In this work, we focused on the whole process in onestage dispersion polymerization and tracked the size evolution of colloidal sphere at different reaction time. Based on the experimental results, we established a theoretical model to describe the relationship between the monomer concentration and the reaction time during dispersion polymerization. The model was expected to provide guidance to the twostage or the multi-stage dispersion polymerization, aiming to synthesize micron-sized large and monodisperse polymer colloidal spheres.

\section{Materials and Methods}

2.1. Materials. All chemicals, including styrene (St, 99\%, Aldrich), anhydrous ethanol (99.95\%, Aldrich), polyvinylpyrrolidone (PVP, MW 40000, TCI), and azobis(isobutyronitrile) (AIBN, Sinopharm Chemical Reagent Co., Ltd), were used as received without further purification. Deionized (DI) water (resistivity greater than $18.2 \mathrm{M} \Omega \cdot \mathrm{cm}$, Ultra Pure UV, China) was used in all experiments.

2.2. Synthesis of PS Colloidal Microspheres by Using Dispersion Polymerization. Briefly, the synthesis was conducted in a three-neck round-bottom flask equipped with a condenser and immersed in a water bath with a preset temperature. Firstly, the monomer of $17 \mathrm{~mL}$ styrene and the stabilizer of $1.5 \mathrm{~g}$ PVP were dissolved in $98 \mathrm{~mL}$ anhydrous ethanol and then added to the flask. The solution was stirred with a rate of $200 \mathrm{rpm}$ and the temperature was kept at $70^{\circ} \mathrm{C}$ while nitrogen gas was continuously led into the flask to avoid the oxidation of the initiator AIBN in the whole process. After 30 minutes, an initiator solution of $0.15 \mathrm{~g}$ AIBN dissolved in $28 \mathrm{~mL}$ ethanol was introduced into the system. The reaction continued for $24 \mathrm{~h}$ and then was stopped by cooling at ambient conditions. To track the evolution of sphere size during dispersion polymerization, the mixture in the reaction flask was sampled at specific intervals and dried on a glass substrate. The sphere size was measured using an optical microscope.

2.3. Characterization. The optical microphotographs of the PS colloidal microspheres were taken using an optical microscope (Olympus, BX51TRF), which was connected to a CCD camera (Pixelink-B742) and a computer for real-time image recording.

\section{Results and Discussion}

3.1. The Morphology of PS Colloidal Spheres Prepared by Dispersion Polymerization. As can be seen from the optical microscope photograph shown in Figure 1, micron-size PS colloidal spheres were obtained by using the one-stage dispersion polymerization method. The PS colloidal spheres were spontaneously organized into a closely packed hexagonal lattice on the glass substrate upon evaporation of solvent, indicating the high monodispersity of the PS spheres. To reduce the error in the measurement of the size of a single PS sphere, we chose the closely packed area and measured the direct distance between the several tangent microspheres in line and calculated the average distance between neighboring two spheres. According to the measured data in Figure 1, the average diameter of the resultant PS colloidal spheres was calculated to be $2.72 \mu \mathrm{m}$.

3.2. Growth Kinetics of PS Spheres during Dispersion Polymerization. The process of dispersion polymerization can be basically divided into several stages. At the beginning of the reaction, monomer, dispersing agent, and initiator are all dissolved in the solvent. When the temperature reaches the decomposition temperature of the initiator, the initiator decomposes and produces radicals. The radicals trigger the polymerization process, which results in formation of the oligomers. When the chain length of the oligomers exceeds a critical value, nucleation occurs and the oligomers precipitate from the solvent. At the same time, dispersing agents are absorbed by the nuclei and form graft copolymers with the polymer chains, which makes microspheres stable in the solvent. Then the synthesis enters the stage of growth. Monomer and copolymer in the solvent are persistently captured by polymer nuclei to provide the motive power of the growth, which keeps on until termination of the reaction. 


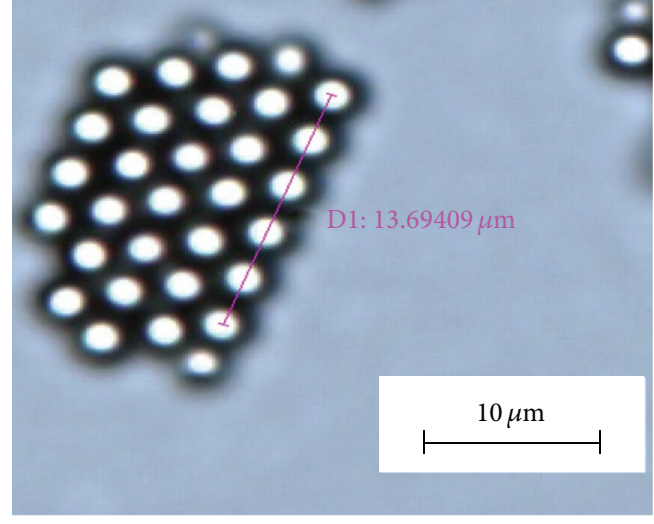

FIGURE 1: Optical microphotograph of PS colloidal spheres prepared by dispersion polymerization.

In the process of dispersion polymerization, the nucleation stage is a transition period for the system from homogenous phase to heterogeneous phase. This period determines the number of microspheres in the system which should be constant during the polymerization to guarantee the uniformity of final microspheres [21, 22, 26-28].

Knowing the evolution of monomer concentration during dispersion polymerization is of importance especially for synthesis of large and uniform colloidal spheres. Considering the reaction mechanism involved in dispersion polymerization, however, a large proportion of the monomers exist in the solvent in the form of oligomers whose chain length does not exceed the critical value for nucleation. Thus, direct measurement of the monomer concentration is not feasible. Herein, we establish a theoretical model to describe monomer concentration at different reaction time based on the diameter of microspheres measured at different time intervals.

The following are the major assumptions used in formulating the model. Firstly, in view of the good monodispersity of the final microspheres, the reaction is considered as an ideal dispersion polymerization, in which nucleation number is just determined by the nucleation stage, and secondary nucleation does not occur in the later growth stage. It is also assumed that the number of microspheres remains during growth stage equal to the nucleation number at the beginning of reaction. Secondly, although the microspheres in the growth stage may mostly seize the oligomer chains rather than the monomers, it is considered that the increasing rate of the microspheres' weight or volume is proportional to the equivalent concentration of the monomers, some of which are in the form of free oligomers. Thirdly, considering the actual slight volume change during the mixture process of styrene and ethanol, which can hardly be observed in the experiment, the deviation of volume is neglected in our model to reduce the complexity of the calculation. Thus, a simple approximation was adopted by the replacement of the total volume of the mixture of several liquids with the sum of their own volumes.
According to the conservation of mass, the relationship between the product and the reactant at any time $t$ is given by

$$
N \rho_{\text {sphere }} V_{\text {sphere }}=m_{0}-C V,
$$

where $N$ is the number of microspheres in the system at time $t, \rho_{\text {sphere }}$ is the density of polystyrene microspheres, $m_{0}$ is the initial mass of styrene monomer added into the system, $C$ is the present concentration of surplus styrene in the system, and $V$ is the total volume of the system at time $t$. $V_{\text {sphere }}$, the average volume of one sphere, can be described by the formula of spheroidal volume

$$
V_{\text {sphere }}=\frac{\pi}{6} d^{3},
$$

where $d$ is the average diameter of microspheres at time $t$.

The concentration of styrene in the system at time $t$ can be expressed using

$$
C=\frac{\rho_{\text {styrene }} V_{\text {styrene }}}{V}=\frac{\rho_{\text {styrene }}\left(V-V_{\text {ethanol }}\right)}{V},
$$

where $V_{\text {styrene }}$ stands for the surplus volume of styrene in the system, while $V_{\text {ethanol }}$ stands for the surplus volume of ethanol, which is equal to the initial volume with the loss in the reaction ignored.

By putting together the three equations above, out comes the relationship between the concentration of monomer and the average diameter of microspheres at time $t$, which can be given by

$$
C=\frac{\rho_{\text {styrene }}\left(m_{0}-N \rho_{\text {sphere }} \cdot(\pi / 6) d^{3}\right)}{m_{0}-N \rho_{\text {sphere }} \cdot(\pi / 6) d^{3}+\rho_{\text {styrene }} V_{\text {ethanol }}} .
$$

Based on our experimental data, we input relevant parameters. The initial parameters were the same as the data described in the experimental section, while the density of microspheres was taken as that of polystyrene. According to the former assumption, the number of microspheres was a constant value which is equal to the nucleation number. Thus, we set the time $t$ at the end of the reaction with the microspheres' diameter $d$ taken as the final size which was $2.72 \mu \mathrm{m}$ in our work, while the final concentration $C_{\text {final }}$ was assumed to be one percent of the initial value. In this way, the number of microspheres was approximately calculated as follows:

$$
N=\frac{\rho_{\text {styrene }} V_{0}-C_{\text {final }} V_{\text {ethanol }}}{\rho_{\text {sphere }} \cdot(\pi / 6) d_{\text {final }}^{3}} \approx 1.347 \times 10^{12} .
$$

Thus, with the input of the initial parameters and the estimated number of microspheres $N$, the relationship between the monomer concentration and the microspheres diameter at any time was attained.

To introduce the time dimension, we measured the diameter of the PS spheres sampled from the reaction system at different time intervals. According to the relationship expressed in (4), we gained the corresponding monomer concentration, which is shown in Table 1. 
TABLE 1: The sphere diameters and monomer concentration at different time.

\begin{tabular}{lcc}
\hline $\begin{array}{l}\text { Reaction time } \\
(\mathrm{h})\end{array}$ & Sphere diameter $(\mu \mathrm{m})$ & $\begin{array}{c}\text { Monomer } \\
\text { concentration }(\mathrm{g} / \mathrm{mL})\end{array}$ \\
\hline 0 & 0 & 0.105 \\
1 & 1.170 & 0.098 \\
1.5 & 1.364 & 0.094 \\
2 & 1.360 & 0.094 \\
3 & 1.760 & 0.080 \\
4 & 1.960 & 0.069 \\
5 & 2.114 & 0.060 \\
7 & 2.376 & 0.039 \\
9 & 2.410 & 0.036 \\
11 & 2.652 & 0.010 \\
23 & 2.720 & 0.001 \\
\hline
\end{tabular}

In order to further deduce the relationship between the monomer concentration and the reaction time, we calculated different function forms of monomer concentration versus reaction time and processed the data with linear fitting. The results are shown in Figure 2.

As can be seen in Figure 2, the linear fitting of $\ln C$ and $t$ has the largest correlation coefficient of 0.965 . As a consequence, we consider that the natural log of the monomer concentration is linearly associated with the reaction time. In other words, there exists an exponential relationship between the monomer concentration and the reaction time, which can be expressed using

$$
C(t)=C_{0} e^{-k t},
$$

where the initial monomer concentration $C_{0}$ can be attained by the initial parameters in our experiment. Then, a program was written to find optimal value of $k$ which minimized the sum of squared relative errors between the theoretical curve and the experimental points $\left(t_{i}, C_{i}\right)$. That was one value that made the value of $\sum_{i}\left[\left(C_{i}-C\left(t_{i}\right)\right) / C\left(t_{i}\right)\right]^{2}$ minimum. The final result was obtained as follows:

$$
C=0.105 \times e^{-0.10 t}
$$

Combining (4) with (7), the relationship between the colloidal sphere diameter and the reaction time could be derived. The resultant curve is shown in Figure 3 in comparison to the experimental data. As can be seen from Figure 3, the theoretical curve matches the experimental data well.

3.3. Application of the Kinetic Model on Synthesis of Large and Monodisperse Colloidal Spheres via Dispersion Polymerization. Through the deformation of (3), the total volume of the reaction system can be obtained as follows:

$$
V=\frac{\rho_{\text {styrene }} V_{\text {ethanol }}}{\rho_{\text {styrene }}-C} .
$$

TABLE 2: Different monomer feeding rates required at various beginning time of continuous monomer feeding.

\begin{tabular}{lcccc}
\hline$t(\mathrm{~h})$ & $C(\mathrm{~g} / \mathrm{mL})$ & $d C / d t(\mathrm{~g} / \mathrm{mL} / \mathrm{h})$ & $V(\mathrm{~mL})$ & $\Delta V(\mathrm{~mL} / \mathrm{h})$ \\
\hline 1 & 0.0950 & -0.00950 & 140.9 & 1.48 \\
2 & 0.0860 & -0.00860 & 139.4 & 1.32 \\
3 & 0.0778 & -0.00778 & 138.0 & 1.18 \\
4 & 0.0704 & -0.00704 & 136.8 & 1.06 \\
5 & 0.0637 & -0.00637 & 135.7 & 0.95 \\
6 & 0.0576 & -0.00576 & 134.7 & 0.86 \\
7 & 0.0521 & -0.00521 & 133.9 & 0.77 \\
8 & 0.0472 & -0.00472 & 133.1 & 0.69 \\
9 & 0.0427 & -0.00427 & 132.4 & 0.62 \\
10 & 0.0386 & -0.00386 & 131.8 & 0.56
\end{tabular}

$\Delta V$ means the monomer feeding rate needed if the beginning of the continuous monomer feeding is set at time $t$.

With the relationship between the monomer concentration and the reaction time described in (7), the monomer consumption rate at any time of the reaction can be easily deduced by taking the derivative of the monomer concentration with respect to time:

$$
\Delta V=\frac{1}{\rho_{\text {styrene }}} \cdot \frac{d C}{d t} \cdot V
$$

Thus, assuming that the initiator does not lose efficacy during the reaction, the monomer concentration will be held at a constant value if one continuously feeds extra monomer into the system according to the consumption rate described in (9). This will be helpful to keep the system at a high reaction rate, leading to the enlargement of the diameter of the final PS colloidal microspheres. In fact, the initiator efficiency will inevitably descend during the process of dispersion polymerization; so in actual process, we can meet the requirement of constant efficiency by replenishing initiator during the reaction.

According to the analysis above, we calculated the relationship between the beginning time of the monomer feeding and the monomer feeding rate within the present system of our experiment, which is shown in Table 2. In this way, even if an extra monomer is added into the reaction system, the monomer concentration will still be low enough to avoid new nucleation as long as one begins the continuous feeding of extra monomer in the growth stage rather in the nucleation state. As a consequence, the monodispersity of the colloidal spheres is ensured. At the same time, continuous feeding of extra monomer inevitably leads to colloidal spheres with large size.

\section{Conclusion}

In summary, a kinetic model has been established to describe the exponential relationship between the monomer concentration and the reaction time during synthesis of colloidal spheres by using dispersion polymerization. Accordingly, one may gain the quantitative relationship between the 


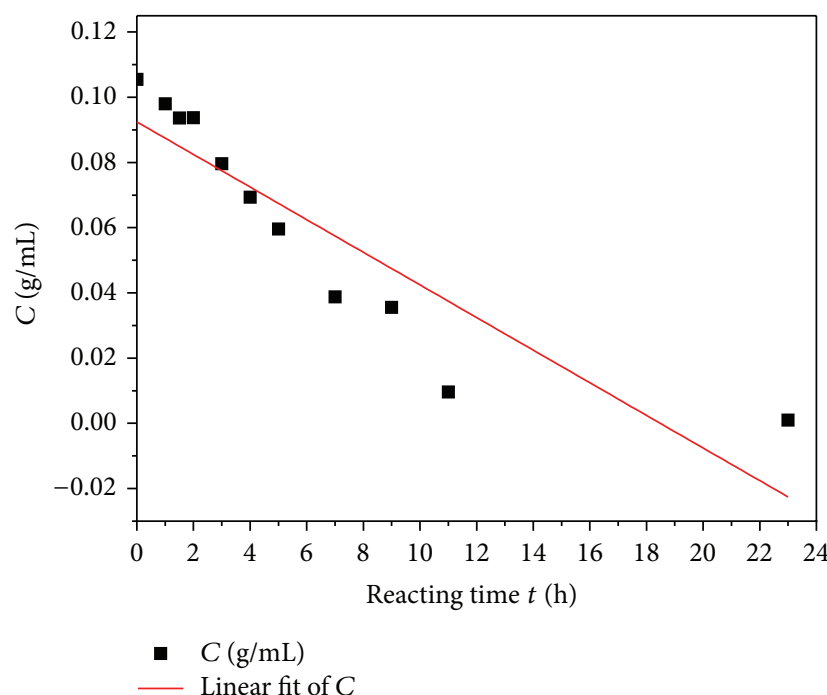

(a)

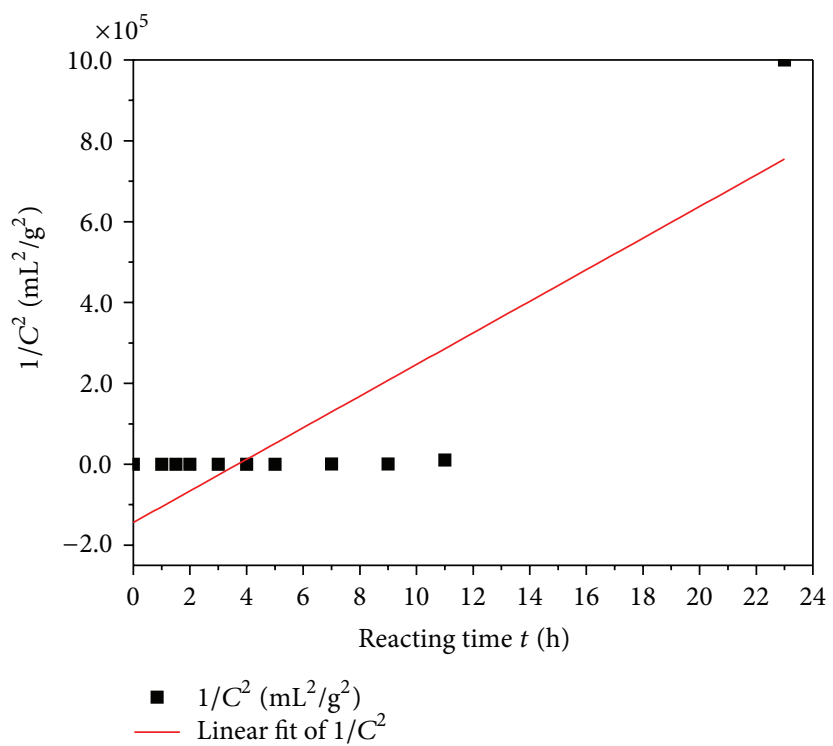

(c)

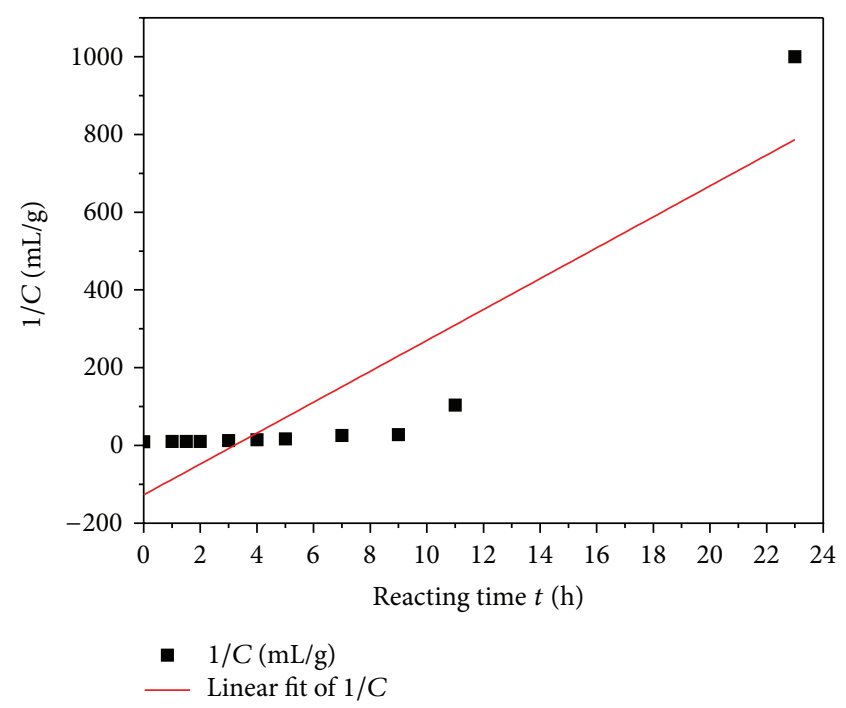

(b)

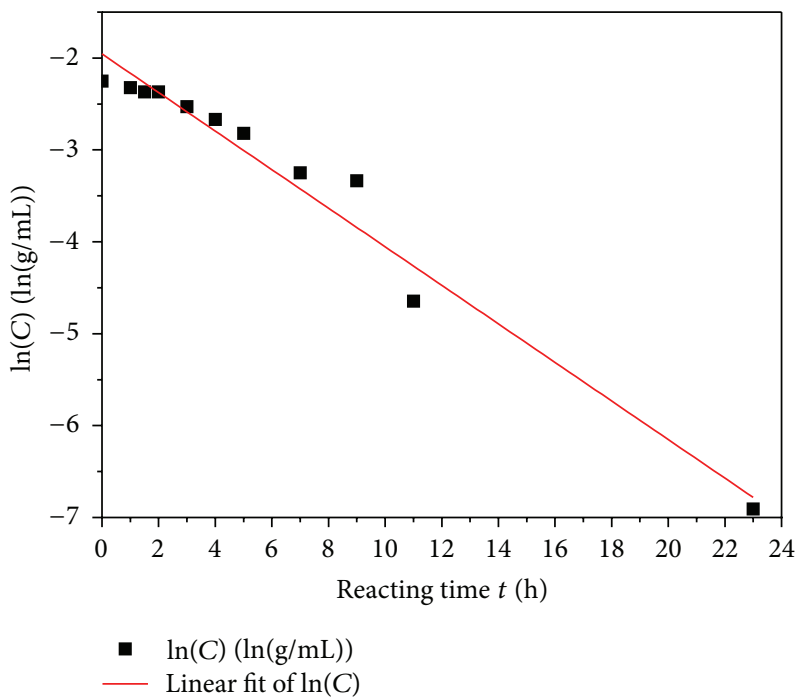

(d)

FIGURE 2: Linear fitting results of different function forms of concentration $C$ with reaction time $t$. (a) linear fitting of $C$ and $t, r=0.800$ (b) linear fitting of $1 / C$ and $t, r=0.763$ (c) linear fitting of $1 / C^{2}$ and $t, r=0.703$ (d) linear fitting of $\ln C$ and $t, r=0.965$.

monomer consumption rate and the reaction time. Taking advantage of this model, it is possible to attain larger colloidal spheres by continuously feeding monomer into the reaction system during the growth stage according to the monomer consumption rate. In this way, the reaction rate is kept at a high constant all the time until the reaction is artificially ended. Furthermore, secondary nucleation can be effectively avoided because both the average concentration and the local concentration of monomer in the reaction system are less than the critical value. Ultimately, large and uniform colloidal spheres can be prepared using the dispersion polymerization technique.

\section{Conflict of Interests}

The authors declare no competing financial interests.

\section{Acknowledgments}

This work was supported by the Natural Science Foundation of China (Project nos. 50903046 and 51173097) and the National Key Basic Research Program of China (no. 2013CB632902). The Tsinghua University Initiative Scientific Research Program and the Tsinghua National Laboratory 


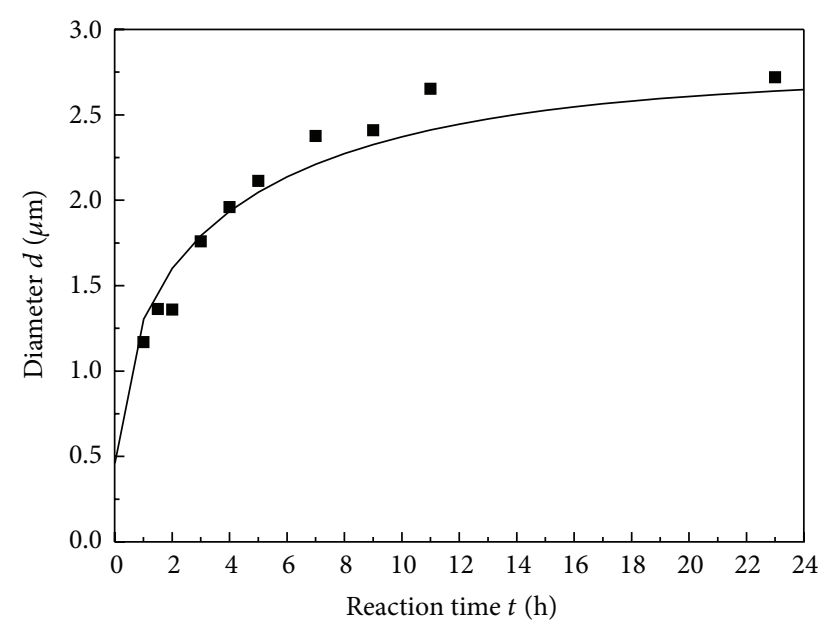

FIGURE 3: The evolution of spheres diameter with extended reaction time. The squares stand for the experimental points.

for Information Science and Technology (TNList) Crossdiscipline Foundation are also acknowledged for partial financial support.

\section{References}

[1] S. K. Mi, H. L. Gyu, H. Jae-Min, and L. Hyunjung, "Synthesis of monodisperse PS-co-PDMS microspheres by dispersion polymerization," Materials Science and Engineering, vol. 27, no. 5-8, pp. 1247-1251, 2007.

[2] S. O. Kyoung, B. won, and K. Hwayoung, "Dispersion polymerization of N-vinylcarbazole using siloxane-based and fluorinebased surfactants in compressed liquid dimethyl ether," Polymer, vol. 48, no. 6, pp. 1450-1454, 2007.

[3] I. Miraballes-Martínez and J. Forcada, "Synthesis of latex particles with surface amino groups," Journal of Polymer Science A, vol. 38, pp. 4230-4237, 2000.

[4] R. P. A. Dullens, E. M. Claesson, and W. K. Kegel, "Preparation and properties of cross-linked fluorescent poly(methyl methacrylate) latex colloids," Langmuir, vol. 20, no. 3, pp. 658664, 2004.

[5] H. Kawaguchi, "Functional polymer microspheres," Progress in Polymer Science, vol. 25, no. 8, pp. 1171-1210, 2000.

[6] R. M. Santos and J. Forcada, "Acetal-functionalized polymer particles useful for immunoassays," Journal of Polymer Science $A$, vol. 35, no. 9, pp. 1605-1610, 1997.

[7] K. Ishii, "Synthesis of microgels and their application to coatings," Colloids and Surfaces A, vol. 153, no. 1-3, pp. 591-595, 1999.

[8] E. Caro, R. M. Marce, P. A. G. Cormack, D. C. Sherrington, and F. Borrull, "Molecularly imprinted solid-phase extraction of naphthalene sulfonates from water," Journal of Chromatography A, vol. 1047, no. 2, pp. 175-180, 2004.

[9] Y. Watebe, K. Hosoya, N. Tanaka, and T. Kubo, "Shielded molecularly imprinted polymers prepared with a selective surface modification," Journal of Polymer Science A, vol. 43, no. 10, pp. 2048-2060, 2005.

[10] S. Omi, M. Saito, T. Hashimoto, M. Nagai, and G.-H. Ma, "Preparation of monodisperse polystyrene spheres incorporating polyimide prepolymer by dispersion polymerization in the presence of L-ascorbic acid," Journal of Applied Polymer Science, vol. 68, no. 6, pp. 897-907, 1998.

[11] K. Ishizu and N. Tahara, "Microsphere synthesis by emulsion copolymerization of methyl methacrylate with poly(methacrylic acid) macromonomers," Polymer, vol. 37, no. 13, pp. 28532856, 1996.

[12] N. B. Viswanthan, P. A. Thomas, J. K. Pandit, M. G. Kulkarni, and R. A. Mashelkar, "Preparation of non-porous microspheres with high entrapment efficiency of proteins by a (water-in-oil)in-oil emulsion technique," Journal of Controlled Release, vol. 58, no. 1, pp. 9-20, 1999.

[13] J. W. Vanderhoff and E. B. Brandford, Polymer Colloid I, Plenum Press, New York, NY, USA, 1971.

[14] D.-H. Lee, J.-W. Kim, and K.-D. Suh, "Monodisperse micronsized polymethylmethacrylate particles having a crosslinked network structure. V,' Journal of Materials Science, vol. 35, no. 24, pp. 6181-6188, 2000.

[15] M. Okubo, M. Shiozaki, M. Tsujihiro, and Y. Tsukuda, "Preparation of micron-size monodisperse polymer particles by seeded polymerization utilizing the dynamic monomer swelling method," Colloid \& Polymer Science, vol. 269, no. 3, pp. 222-226, 1991.

[16] J. Ugelstad, P. C. Mork, R. Schmid, T. Ellingsen, and A. Berge, "Preparation and biochemical and biomedical applications of new monosized polymer particles," Polymer International, vol. 30, no. 2, pp. 157-168, 1993.

[17] C.-M. Tseng, M. S. El-Aasser, and J. W. Vanderhoff, “Optimization of polymer-solvent interaction in a multisolvent system by the unifac group-contribution method," Journal of Applied Polymer Science, vol. 32, no. 5, pp. 5007-5019, 1986.

[18] H. Bamnolker, B. Nitzan, T. Tennenbaum, B. BarToov, M. Hinz, and H. Seliger, "Scientific and Clinical Applications of Magnetic Carriers ", in Proceedings of the 1st International Conference on Scientific and Clinical Applications of Magnetic Carriers, pp. 3751, Rostock, Germany, September 1996.

[19] A. Tuncel, R. Kahraman, and E. Piskin, "Monosize polystyrene lattices carrying functional-groups on their surfaces," Journal of Applied Polymer Science, vol. 51, pp. 1485-1498, 1994.

[20] K. Hu, V. L. Dimonie, E. D. Sudol, and M. S. El-Aasser, "Monodisperse poly(butadiene/styrene) particles by dispersion polymerization," Journal of Applied Polymer Science, vol. 55, no. 10, pp. 1411-1415, 1995.

[21] B. Thomson, A. Rudin, and G. Lajoie, "Dispersion copolymerization of styrene and divinylbenzene: synthesis of monodisperse, uniformly crosslinked particles," Journal of Polymer Science A, vol. 33, no. 3, pp. 345-347, 1995.

[22] B. Thomson, A. Rudin, and G. Lajoie, "Dispersion copolymerization of styrene and divinylbenzene. II: effect of crosslinker on particle morphology," Journal of Applied Polymer Science, vol. 59, no. 13, pp. 2009-2028, 1996.

[23] M. Holderle, G. Bar, and R. Mulhaupt, "Synthesis and characterization of oxazoline-functional polymer particles by free radical nonaqueous dispersion polymerization," Journal of Polymer Science A, vol. 35, no. 13, pp. 2539-2548.

[24] D. Horak, F. Svec, and J. M. Frechet, "Preparation and control of surface properties of monodisperse micrometer size beads by dispersion copolymerization of styrene and butyl methacrylate in polar media," Journal of Polymer Science A, vol. 33, no. 14, pp. 2329-2338, 1995. 
[25] J.-S. Song, F. Tronc, and M. A. Winnik, “Two-stage dispersion polymerization toward monodisperse, controlled micrometersized copolymer particles," Journal of the American Chemical Society, vol. 126, no. 21, pp. 6562-6563, 2004.

[26] H. Kurioka, H. Uyama, and S. Kobayashi, "Peroxidase-catalyzed dispersion polymerization of phenol derivatives," Polymer Journal, vol. 30, no. 6, pp. 526-529, 1998.

[27] M. Okubo, T. Yamashita, and E. Ise, "Thermodynamics for preparation of micron-sized, monodispersed, monomeradsorbed polymer particles having snowman shape by utilizing the dynamic swelling method and the seeded polymerization," Proceedings of the Japan Academy B, vol. 75, no. 7, pp. 195-200, 1999.

[28] M. Okubo, Y. Konishi, M. Takebe, and H. Minami, "Preparation of micron-sized, monodispersed,anomalous polymer particles by utilizing the solvent-absorbing/releasing method," Colloid and Polymer Science, vol. 278, no. 10, pp. 919-926, 2000. 

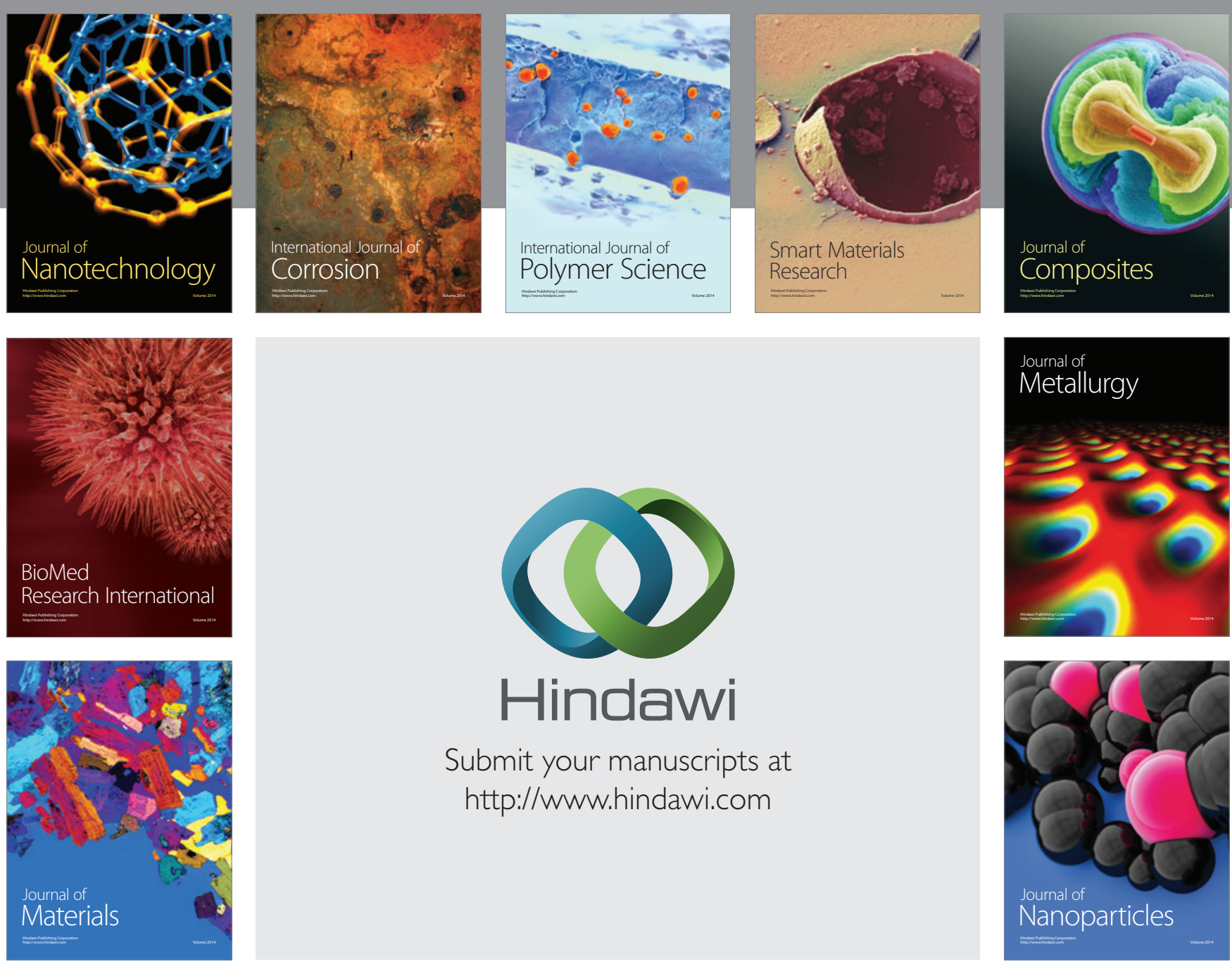

Submit your manuscripts at http://www.hindawi.com
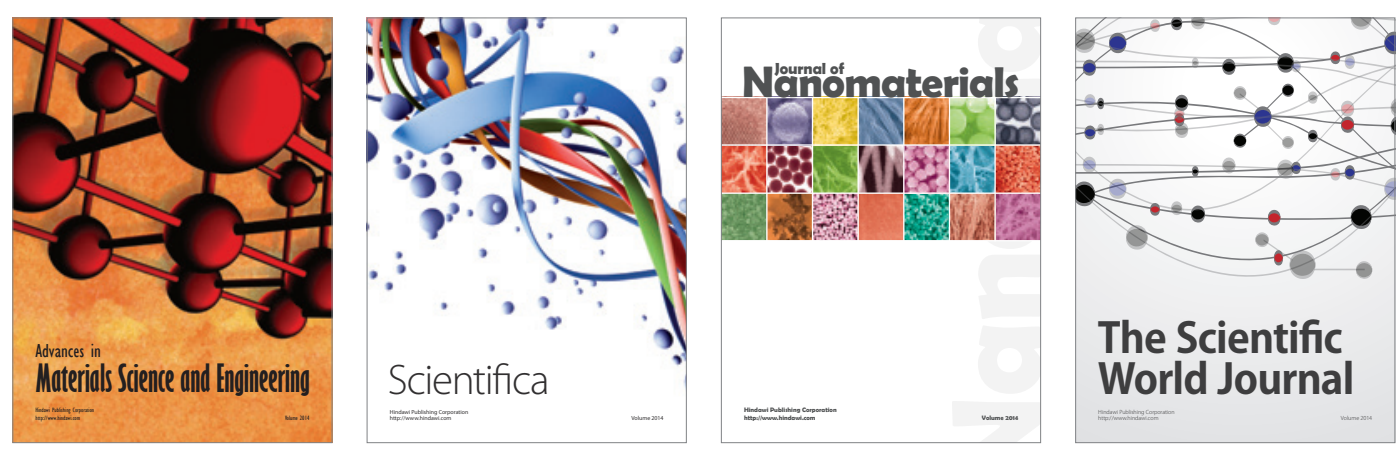

\section{The Scientific World Journal}
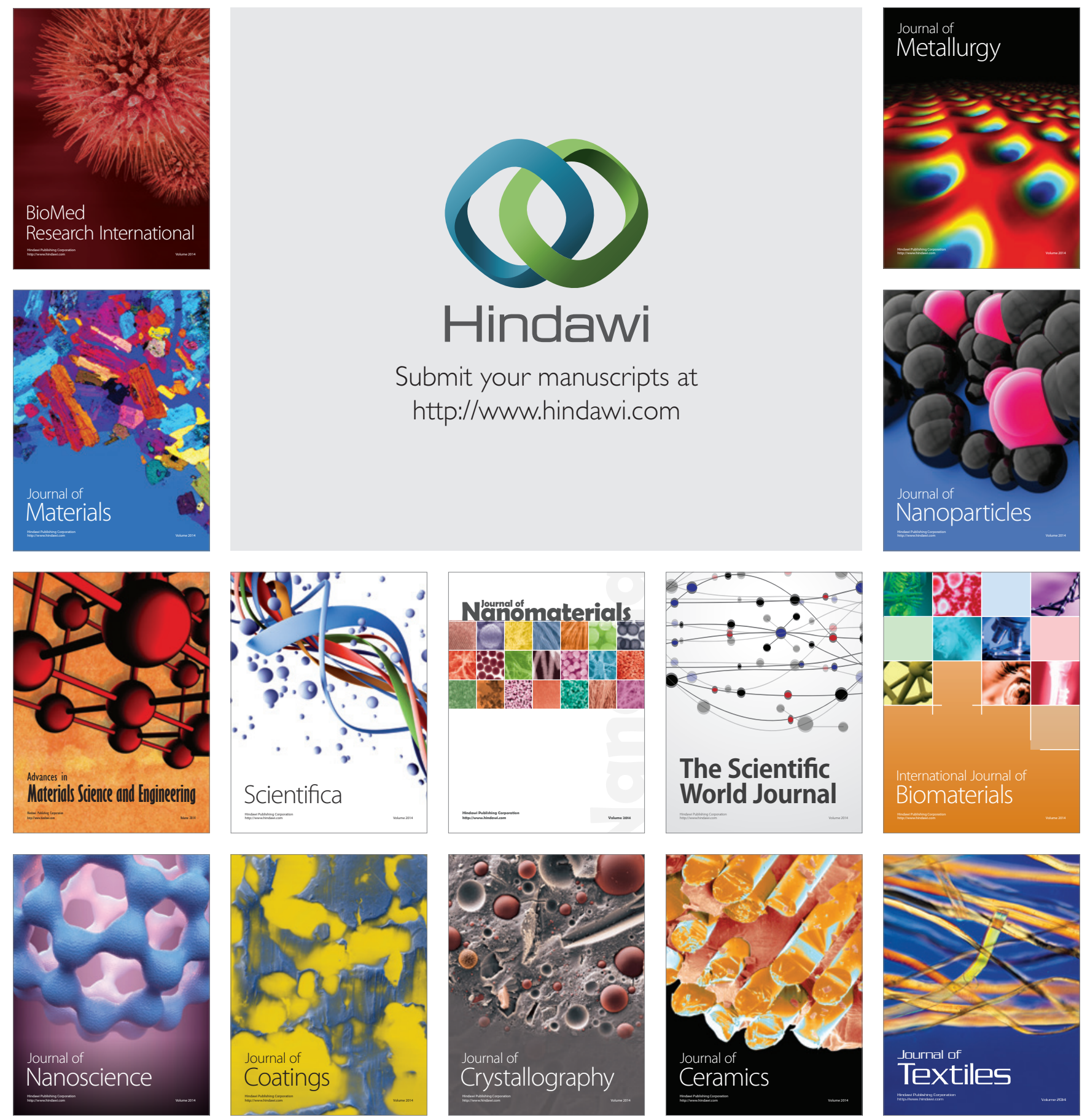